\title{
Design of the CCD Color Selector
}

\author{
Dacheng Sun \& Qirui Han \\ School of Electrical Engineering \& Automatic, Tianjin Polytechnic University \\ Tianjin 300160, China \\ E-mail: linda-dzxy@163.com
}

\begin{abstract}
The color selector is the quick food selection equipment integrating many high technologies such as optics, machine and electrics, and it is mainly used to separate heterochromous grains such as yellowing grains, mildew grains and impurities from rice, peanuts, melon seeds and sesames, and it is the important equipment to ensure the safety and quality of grains. In this article, the design principle and the composing parts of the CCD color selector are simply introduced. The color selector includes the main control system, the checking separation system, the vibration feed-in system and the optical system. The main control system mainly controls and manages various subsystems, and harmonizes exterior functional modules. The checking separation system mainly selects and eliminates the heterochromous grains checked by the optimal system. The vibration feed-in system is used to control the flux of materials. The optical system is mainly composed by the optical illumination system and the optical imaging system, and it is the core part of the color selector.
\end{abstract}

Keywords: CCD, FPGA, $\mu$ C/OS-II, MODBUD

\section{Research meanings and actuality of the CCD color selector}

China is a large agricultural country which needs to provide high-quality and safe food for one fifth population of the world, and its grain processing industry is very important in the agricultural industrialization course. With the enhancement of people's living level, their requirements to the food safety are higher and higher, which directly promotes the development of the color selector in China. The application of the color selector first developed in the rice industry, and the color selector in the rice manufacturing factory which produces refined rice is mainly used to select the yellowing rice, red rice, paunch-white rice, mildew rice, black scab rice, sands clods and other heterochromous impurities. After selected and purified by the color selector, the intact, pure rice grains with high transparence and breed purity and intrinsic normal color and luster can be picked out, so the quality of the rice will be significantly enhanced. Except for that, the color selector is also developed quiet greatly in the seeds, oil manufactures and fruits, and the prices of many senior food materials are increased significantly after the selection by the color selector.

Foreign companies studied the color selectors early. US ESM Company and Sortxe Company researched this equipment respectively in 1930s and 1940s, and they continually pushed new products according to the market demands after that. In 1979, Satake Corporation of Japan first pushed this product, and at present the GS series rice color selector produced by it has excellent performance, and the relative technical indexes are stable, and it has been applied widely. Since Japan Anza Company was founded, it applied itself to the research about the photoelectric separation technology, and in April of 2002, it successfully developed the special selector which was used to select the low-viscosity wheat, and in the same year, it developed and produced the large-scale color selector carrying high-speed CCD.

At present, there are mainly Japanese, Korea and Italian products in the Chinese market of the color selector, and most of them belong to the electro optical selector, and a few of them are CCD color selector. Comparing with foreign enterprises, domestic enterprises are in the stage of $R \& D$ or batch production, and there are a few molding products, and the total technology and technical manufacturing level are bad, and the national standards have not been established. The current color selectors in the domestic market mainly includes the selector series made by China-US Joint Venture Hefei An Ke Optoelectronic Technology Co., Ltd which introduced US technical production, and the selector independently researched and produced by the Research Institute of Physical and Chemical Engineering of Nuclear Industry which has been authenticated by China. But there are many parts which should be improved in the structure design of the domestic selector, and various technical indexes have certain gaps with foreign products'.

In a word, whether from the technical development or from the market demand, the research about the optoelectronic color selector is very meaningful. 


\section{Working principle and structure of the CCD color selector}

The common selection technology is to use the mechanical equipment to eliminate impurities, and its main principle is to utilize the differences of the size, shape or density and other physical features between impurities and grains to implement the selection. But the working principle of the optoelectronic color selector is to utilize the different features of the optical induction intension between impurities and grains to implement the selection. Taking the color selection of rice as the example, before the rice are selected by the color selector, the color selection mode, output and other parameters should be set up first according to the proportion and species of the impurities in the material rice. Then the materials are sent to the material vibrator, and enter into the chute with certain angle by the vibrator, and the optical cameras installed at two sides of the color selector performs the illumination comparison to catch the materials and impurities which are differently colorful with the eligible materials, and then the selector translates the optical signals into the electric signals, and orders the magnetic valve to start the high-pressure airflow muzzle to blow away the objects, so the disqualified materials and impurities are rejects. After the first-time selection is accomplished, the materials are transferred to the hopper to implement the second-time color selection, and the qualified rice directly enters into the next working procedure. The disqualified rice selected by the second-time color selection channel is stored in the disqualified rice hopper. Figure 1 is the principle chart of the electro optical color selector.

\section{Main parts of the CCD color selector}

The CCD color selector mainly includes the main control system, the checking separation system, the vibration feed-in system and the optical system. The main control system adopts the C8051F040 SCM made by the New China Dragon Electronic Co., Ltd as the controller which embeds the $\mu \mathrm{C} / \mathrm{OS}$-II operation system to ensure the stable running of the whole system, and use the DMT320240_11 touch screen made by the Beijing Dwin Technology Co, Ltd as the touch screen. The checking separation system uses the EP2C5Q208C of the ALTERA Company to implement the processing of the high-speed signals collected by CCD. The vibration feed-in system adopts the electromagnetic vibration mode to design the control system based on the C8051F320 SCM which includes the zero-crossing detector and the quadruple controlled silicon trigger circuit. The communication circuits between the main control system and the touch screen and between the main control system and various systems are designed in the article to implement the RS-232 serial communication connection between the main control system and the touch screen and the asynchronous serial communication connection based on the MODBUS protocol among various subsystems.

\subsection{Design of the main control system}

The main control system uses the communication interface circuit to connect the touch screen, the selection control board, the electromagnetic liberation feed-in system, the electronic control board, the front background board motor and the back background board motor. The C8051F040 SCM of New China Dragon Electronic Co., Ltd is used as the controller of the main control system, and its running speed is quick, and the feet input and output drive ability is strong, and the exterior functions are abundant, and the power consumption is low, and the running is stable, and the price is proper, so it is selected as the main control chip of the CCD color selector. Figure 1 is the total flow of the main control system.

As the core part of the whole CCD color selector, the main control system can transfer various subsystems and the communications, so the stable running of the main control system is the key for the stable running of the whole system. To ensure stabilization of the main control system, the embedded real-time operation system $\mu \mathrm{C} / \mathrm{OS}-\mathrm{II}$ is adopted. The $\mu \mathrm{C} / \mathrm{OS}$-II system is offered by the Micrium Corporation, and it is a transplantable, solidified, cut and anticipated multi-task real-time kernel, and it is applied in the micro-processor, micro-controller and the digital processing chip. At the same time, the source codes of the system are opening, neat and consistent, and the explanation is detailed, and it is fit for the development of the system. The $\mu \mathrm{C} / \mathrm{OS}-\mathrm{II}$ system has been authenticated by the standards of the commercial aircraft of Federal Aviation Administration (FAA), according with the standard of DO-178B of Radio Technical Commission for Aeronautics (RTCA). The commercial applications of the $\mu \mathrm{C} / \mathrm{OS}$-II real-time system are very extensive, and the system has very stable and reliable performances, and has been successfully applied in many R\&D projects such as life science and spaceflight engineering. Because of its tiny kernel, it is the same with the embedded system development which requires very sensitive program code memory space especially. The $\mu \mathrm{C} / \mathrm{OS}-\mathrm{II}$ system is transplanted into the C8051F040 SCM, and it occupies about 1k ROM space and about 1k RAM space. And the C8051F040 SCM contains 64k Flash and 4k of RAM, and fulfills the requirements of the $\mu \mathrm{C} / \mathrm{OS}$-II system. The facts have proved that after the $\mu \mathrm{C} / \mathrm{OS}-\mathrm{II}$ system was adopted, the stability of the whole CCD color selector was further enhanced.

The communications of various subsystems of the CCD color selector mainly depend on the UART port to implement the serial data communication. To ensure the stable operation of the system communication, the communication adopts the MODBUS protocol which has been widely the industrial controller networking. The MODBUS protocol invented in 1978is the communication protocol which is used in control and communication of the electronic controller. By this protocol, the controller uses the network and other equipments to communicate. Its features such as the opening, the 
expansion and the standardization make it become a general industrial standard. The MODBUS protocol adopts the host-slave principles, and if one controller sends one message, it is only the main equipment to anticipate obtaining the response from the equipment. By the same way, when the controller receives one message, it will establish a controller which responses the format from the equipment and returns to the transmission. This "ask-answer" communication form can ensure the stabilization of the whole system communication.

\subsection{Design of the checking separation system}

The checking separation system is the core organ of the whole CCD color selector, and it modulates the signals picked by the optical system through the modulating circuit, and performs many processes such as analog-digital conversion and storage, and then the signals enter into the signal processor to generate the execution signals which are amplified to become the power pulse signals, and finally the system start the drive circuit and the muzzle to achieve the intention of selection. Figure 2 is the flow of the checking separation system.

The checking component of the CCD color selector decides the precision and the sensitivity of the whole system. In the system, the TCD1280AP CCD is adopted as the image sensor. The TCD1280AP has 2160 image sensitive units, and the image unit size and the space between are $14 \mu \mathrm{m} \times 14 \mu \mathrm{m}$. It has many features such as high sensitivity and low dark current, and the work pressure is single $5 \mathrm{~V}$, and it is the line array CCD component with two-phase output. It is mainly used in many situations such as the communication faxes, image scanning and optical character reader. The small signal generated by the TCD1280AP must be amplified and translated through A/D to enter into FPGA for processing.

The checking separation system should process the collected high-speed signals, so the new generation FPGA-Cyclone II of the ALTERA Company with low cost is selected. The FPGA-Cyclone II can not only fulfill the requirements of the system, but reduce the costs. For the engineering design of FPGA, its general development flow includes many approaches such as circuit design and input, function simulation and integration and implementation, the simulation and testing and debugging after locating and wiring. The Quartus II software of the ALTERA Company adopted in the system almost can offer all design flow tools, and it adopts the usual method combining the Verilog hardware description language with the principle chart to design the input method, which can not only divide the design into many parts which are more conveniently managed, but simplify the auditing process of the design. To test whether the circuit function accord with the requirements of the design, the online logic analyzer (SignalTap II) interiorly integrated in QuartusII to implement the simulation of the online system.

\subsection{Design of the liberation feed-in system}

The liberation feed-in system is mainly composed by the liberation feeding instrument which is the core of the material feed-in system, and it is a half-wave controlled commuting excitation instrument. After the commuting function of the diode, AC currents are conversed into the half-wave pulse currents, and when the currents go crossing the electronic magnet, the electronic magnet will generate the magnetism, and the magnetic flux will change continually according o the Lenz's Law, so the function forces between it and the armature will change continually. By the joint function with the resonance reed, the hopper will be liberated and the materials will flow from the hoppers, so the attention of feed-in will be achieved. The liberation feed-in system should run stably, and the material feed-in should not only fulfill the requirements of the output, but can not generate too large fluctuations and impact the test to the materials.

\subsection{Design of the optical system}

\subsubsection{Optical illumination system}

The illumination system is the illumination instrument composed by the light source, the diaphragm, the illuminator and the optical collector. The imaging quality of the optical system is closely related with the illumination of the object. The illumination light is offered by the light source, and it realizes the illumination of the object by the illumination system which is an important part of the optical system. It should equip the illumination system to study invisible objects. The factors that the illumination system impacts the imaging of the optical system are the lightness of the light source, the irradiance area, and the invariable of the illumination system. The confirmations of the light source parameters can be calculated by the luminosity. To offer necessary illumination vision field and aperture, the light pipe composed by the light source and the illumination system must be full of the entrance pupil and the object plane of the subsequent optical system. The adopted collecting illumination system can illuminate the object by the small area light source, which can reduce the size the light source and make the object plane to be closer with the optical collector (seen in Figure 3).

\subsubsection{Optical imaging system}

The collecting light path of the imaging system is mainly divided into the telecentric light path of object space and the telecentric light path of image space (seen in Figure 4). In the telecentric light path of object space, the diaphragm of the aperture is located in the focus of the image space, and here, the incidence pupil is at the infinite place of the object space, and the exit pupil superposes the diaphragm of the aperture. The main function of the telecentric light path of object space is to eliminate or reduce the measurement errors induced by the parallax. In the telecentric light path of 
image space, the diaphragm of the aperture is located at the focus of the object space, and here, the emergence pupil is at the infinite place of the image space, and the entrance pupil superposes with the diaphragm of the aperture, and the main function of the telecentric light path of the image space is to eliminate or reduce the measurement error.

\section{Conclusions}

The system is mainly composed by the line-array CCD, MCU and FPGA. After the input materials are illuminated by the light source, they are imaged on the array of the CCD photosensitive components by the object lens, and CCD accomplishes the scanning once by the driver circuit. Under the function of the control circuit, the signals outputted by $\mathrm{CCD}$ are amplified through the filtering waves, and enter into FPGA to implement the digitalized processing by the A/D circuit, so the corresponding execution signals are generated to drive the electromagnetic valve to push the impurities. Above operations are performed in phase with the CCD scanning, free of the control of MCU which only harmonizes the running of various subsystems.

In a word, the electro optical selector is a very complex machine which requires high precisions, and there are many key technologies which should be studied in the future.

\section{References}

Chen, Yaohua. (1996). Checking Neps in Nankeens by CCD Camera. Transactions of the Chinese Society of Agricultural Engineering. No.3.

Chen, Zaiping. (2008). CAN-bus and Industrial Control Network Technology. Beijing: Electronic Industry Press.

Han, Bing \& Yu, Fei. (2006). Application Examples of the CAN-bus Control System. Beijing: Chemical Industry Press.

Jean J. Labrosse. (2001). Embedded Operation System $\mu$ C/OS- II. Beijing: Beijing Aviation and Spaceflight University Press.

Tong, Fei. (2005). C8051F SCM Development and C-Language Programming. Beijing: Beijing Aviation and Spaceflight University Press.

Wang, Cheng. (2001). Design of Altera EPGA/CPLD. Beijing: Renmin Posts \& Telecom Press.

Wang, Qingyou. (2000). Application Technology of CCD. Tianjin: Tianjin University Press.

Wang, Qingyou \& Sun, Xuezhu. (1993). CCD Application Technology. Tianjin: Tianjin University Press.

Xia, Yuwen. (2003). Tutorial of Verilog Digital System Design. Beijing: Beijing Aviation and Spaceflight University Press.

Zhang, Lin. (1997). Electro Optical Selector and Its Application. Machinery for Cereals Oil and Food Processing Main Contents. No.2.

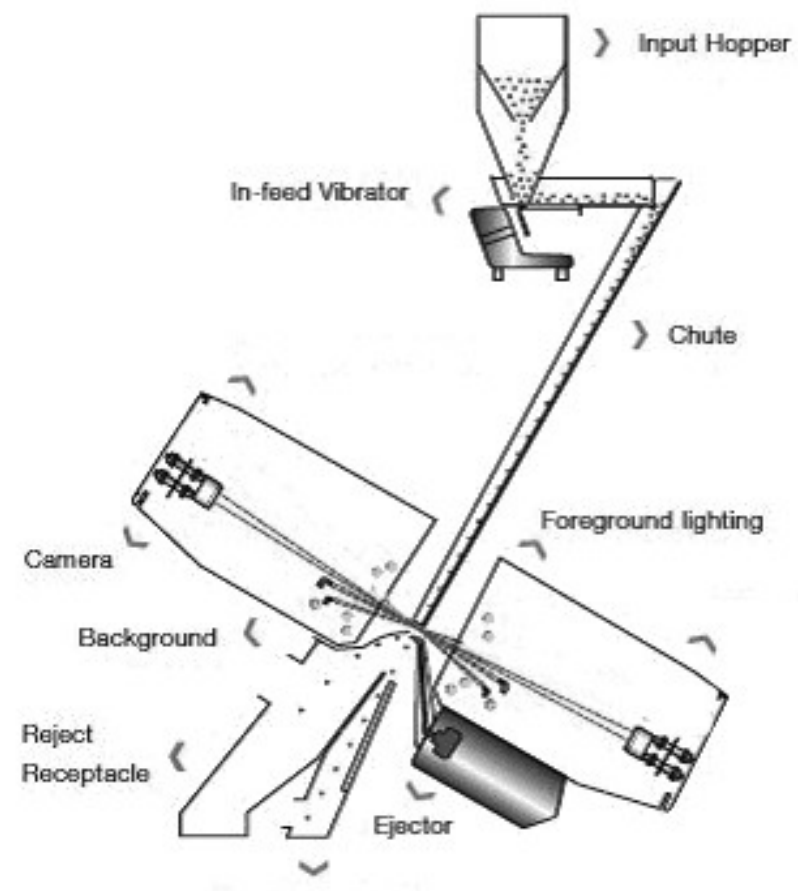

Figure 1. Principle of the Electro Optical Selector 


\begin{tabular}{|c|c|c|c|c|c|}
\hline $\begin{array}{l}\text { Photoelectric } \\
\text { pulse signals }\end{array}$ & $\begin{array}{l}\text { Modeling } \\
\text { and } \\
\text { Amplifying }\end{array}$ & $\begin{array}{l}\text { Judging and } \\
\text { identifying }\end{array}$ & $\begin{array}{l}\text { Power } \\
\text { pulse } \\
\text { signals }\end{array}$ & $\begin{array}{l}\text { Drive } \\
\text { circuit }\end{array}$ & Selection \\
\hline
\end{tabular}

Figure 2. Flow of the Checking Separation System

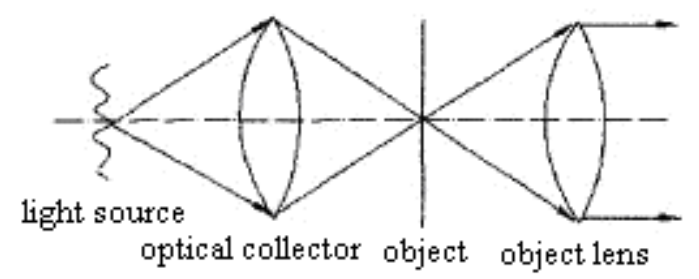

Figure 3. Collecting Illumination System

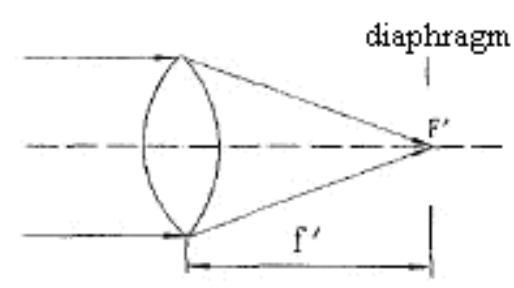

telecentric light path of object space

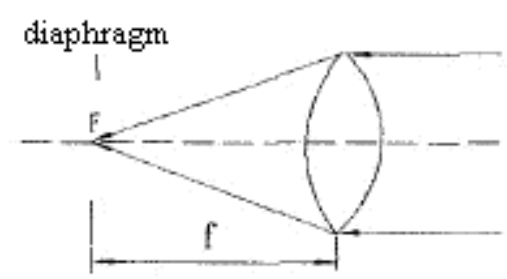

telecentric light path of image space

Figure 4. Telecentric Light Path 